\title{
Probing QCD with Photons and Jets at the ATLAS detector
}

\author{
Giuseppe Callea ${ }^{1, a}$, on behalf of the ATLAS Collaboration \\ ${ }^{1}$ Università della Calabria and INFN-Cosenza
}

\begin{abstract}
This contribution gives an overview of the recent measurements of the differential cross sections for final states involving photons and/or jets at the centre-of-mass energies of 8 and $13 \mathrm{TeV}$, published by the ATLAS Collaboration. The results are compared with several next-to-leading order calculations and with the latest predictions of various Monte Carlo generators. New measurements of transverse energy-energy correlations and their associated asymmetries in multi-jet events at $8 \mathrm{TeV}$ are also presented. Both measurements are used to extract the strong coupling constant and test the renormalization group equation.
\end{abstract}

\section{Introduction}

The study of the production of jets and photons at the Large Hadron Collider (LHC) provides a powerful tool for understanding perturbative QCD (pQCD). The production of prompt photons in protonproton collisions, provides a testing ground for $\mathrm{PQCD}$ in a cleaner enviroment than in jet production, since the colorless photon originates directly from the hard interaction. The prompt photon analyses described in this contribution include both photons coming from the direct contribution and from the fragmentation process [1]. An isolation requirement is applied in order to reduce the background from photons produced during hadronisation. Moreover, the jet production measurements are used to contrain the gluon density in the proton and can be exploited to extract the strong coupling constant $\left(\alpha_{\mathrm{s}}\right)$ in specific final state topologies. This paper presents the recent published measurements of the cross section of final states involving photons and/or jets at center-of-mass energies of 8 and $13 \mathrm{TeV}$ with the data collected by ATLAS detector [2]. We also present a measurement of the transverse energy-energy correlations (TEEC) and their associate asymmetries (ATEEC) in multi-jet events at 8 $\mathrm{TeV}$ and a method to extract $\alpha_{\mathrm{s}}$ from them.

\section{Photon production}

\subsection{Inclusive isolated photon production}

Inclusive isolated-photon production at $13 \mathrm{TeV}$ has been studied using a dataset with an integrated luminosity of $3.2 \mathrm{fb}^{-1}$ [3]. Cross sections as a function of $E_{\mathrm{T}}^{\gamma}$ are measured in four different regions of the photon pseudorapidity $\eta^{\gamma}$ for photons with $E_{\mathrm{T}}^{\gamma}>125 \mathrm{GeV}$ and $\left|\eta^{\gamma}\right|<2.37$. The predictions of the Leading Order (LO) Monte Carlo (MC) generators Pythia [4] and Sherpa [5] give an adequate

\footnotetext{
ae-mail: giuseppe.callea@cern.ch
} 


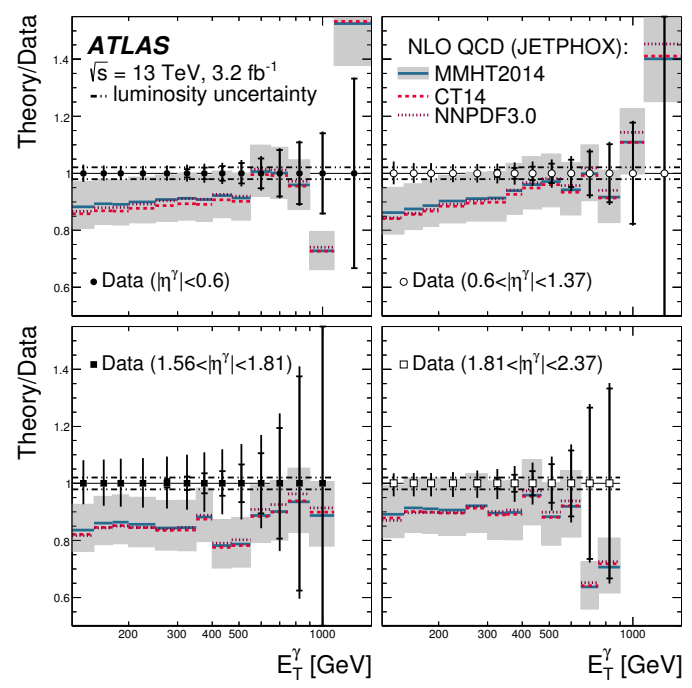

Figure 1. Ratio of the NLO pQCD predictions from JETPHox based on the MMHT2014 PDFs to the measured cross sections for isolated-photon production (solid lines) as a function of $E_{\mathrm{T}}^{\gamma}$ in four $|\eta|$ bins [3]. The black error bars represent the experimental uncertainties, the dot-dashed lines represent the uncertainty due to the luminosity measurement and the shaded bands display the theoretical uncertainty. The ratio of the NLO pQCD predictions based on the CT14 (dashed lines) or NNPDF3.0 (dotted lines) PDF sets to the data are also included.

description of the data, except for $E_{\mathrm{T}}^{\gamma} \geq 500 \mathrm{GeV}$ in the regions of $\left|\eta^{\gamma}\right|<0.6$ and $0.6<\left|\eta^{\gamma}\right|<1.37$. The photon energy is the dominant systematic uncertainty at high $E_{\mathrm{T}}^{\gamma}$ while, at low $E_{\mathrm{T}}^{\gamma}$ the uncertainty related to the background subtraction method dominates. The uncertainties are larger in the $\left|\eta^{\gamma}\right|-$ forward regions. In Figure 1, the NLO pQCD predictions of JETPHox [6], using the MMHT2014 PDF set, are shown, providing an adequate description of the data within the theoretical uncertainties, which are larger than the experimental ones.

\subsection{Photon + jet production}

Measurements of the cross sections for the production of an isolated photon in association with up to three jets have been studied with the ATLAS detector at $8 \mathrm{TeV}$ [7].

The photon is required to have $E_{\mathrm{T}}^{\gamma}>130 \mathrm{GeV}$ and $\left|\eta^{\gamma}\right|<2.37$. The jets are reconstructed using the anti- $\mathrm{k}_{\mathrm{t}}$ algorithm [8] with radius parameter 0.6. The differential cross sections for photon plus one jet are measured as functions of $E_{\mathrm{T}}^{\gamma}$ and $p_{\mathrm{T}}^{\text {jet1 }}$ with $p_{\mathrm{T}}^{\text {jet1 }}>100 \mathrm{GeV}$. For the measurements of the cross sections as a function of $m_{\gamma \text {-jet1 }}$ and the scattering angle in the center-of-mass frame $\left(\left|\cos \theta^{*}\right|\right)$, an additional requirement is imposed: $m_{\gamma \text {-jet1 }}>467 \mathrm{GeV}$. The NLO QCD predictions of JETPHOx, corrected for hadronisation and underlying-event effects, give a good description of the measured cross section distributions in both shape and normalisation. In particular, the measured differential cross section as a function of $\left|\cos \theta^{*}\right|$ and its scale dependence is consistent with the dominance of processes in which a quark is being exchanged.

Photon plus two-jets production is investigated by measuring differential cross sections as functions of $E_{\mathrm{T}}^{\gamma}$ and $p_{\mathrm{T}}^{\text {jet2 }}$ and the azimuthal separation between the final state objects for $p_{\mathrm{T}}^{\text {jet1 }}>100 \mathrm{GeV}$ and $p_{\mathrm{T}}^{\text {jet2 }}>65 \mathrm{GeV}$. The predictions of SHERPA, which include higher-order tree level matrix elements, give 
a better description of $p_{\mathrm{T}}^{\text {jet2 }}$ and the angular distributions, respect to those of PYTHIA, which are based on $2 \rightarrow 2$ processes. The NLO QCD predictions of BLACKHAT [9] provide a good description of the measured differential cross sections, except for $E_{\mathrm{T}}^{\gamma}>750 \mathrm{GeV}$, as shown in Figure 2.

Photon plus three-jets production is characterised by measuring the differential cross sections as
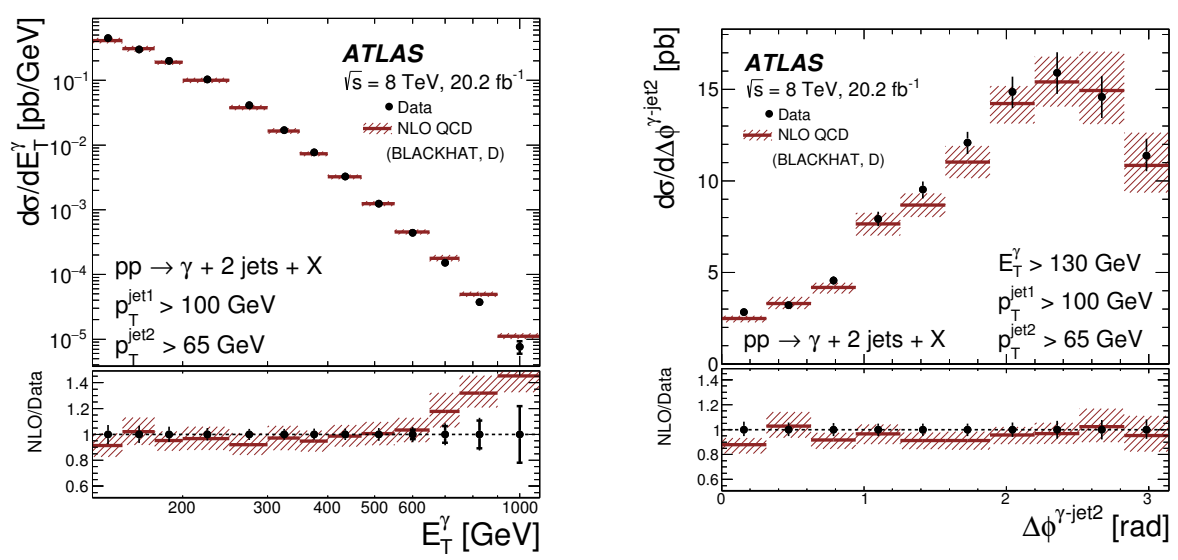

Figure 2. Measured cross section for isolated-photon plus two jets production (dots) as a function of $E_{\mathrm{T}}^{\gamma}$ (left) and of the azimuthal separation between the photon and the sub-leading jet, $\Delta \phi^{\gamma \text {-jet2 }}$, (right) [7]. The NLO QCD predictions of BцаскНАт based on the CT10 PDF set (solid lines) are also included for comparison. The bottom part of both figures shows the ratio of the NLO QCD prediction to the measured cross-section. The black error bars respresent the experimental uncertainties while the shaded bands are the theoretical uncertainties of the calculation.

functions of $E_{\mathrm{T}}^{\gamma}$ and $p_{\mathrm{T}}^{\text {jet3 }}$ and the azimuthal separation between the final state objects for $p_{\mathrm{T}}^{\text {jet1 }}>100$ $\mathrm{GeV}, p_{\mathrm{T}}^{\text {jet2 }}>65 \mathrm{GeV}$ and $p_{\mathrm{T}}^{\text {jet3 }}>50 \mathrm{GeV}$. Sherpa describes better than Pythia $p_{\mathrm{T}}^{\text {jet3 }}$, while both of them give a good description of the angular distributions. The NLO QCD predictions of BLACKHAT provide a good description of the measured differential cross sections.

The dynamics of the isolated-photon production in association with a jet have been also studied using the dataset collected with the ATLAS detector in 2015, with an integrated luminosity of $3.2 \mathrm{fb}^{-1}$ [10]. Photons are required to have $E_{\mathrm{T}}^{\gamma}$ above $125 \mathrm{GeV}$. Jets are reconstructed using the anti- $\mathrm{k}_{\mathrm{t}}$ with radius parameter $\mathrm{R}=0.4$ and are required to have $p_{\mathrm{T}}^{\text {jet }}$ greater than $100 \mathrm{GeV}$ and $\left|y^{\mathrm{jet}}\right|<2.37$. Measurements of isolated-photon plus jet cross sections are presented as functions of the leading photon tranverse energy, the leading jet transverse momentum $p_{\mathrm{T}}^{\text {jet-lead }}$, the azimuthal separation between the photon and the jet, the invariant mass of the photon-jet system and $\left|\cos \theta^{*}\right|$. The differential cross sections as a function of $m^{\gamma \text {-jet }}$ and $\left|\cos \theta^{*}\right|$ are performed in an unbiased phase-space by requiring: $\mid \eta^{\gamma}+$ $y^{\text {jet-lead }}|<2.37,| \cos \theta^{*} \mid<0.83$ and $m^{\gamma \text {-jet }}>450 \mathrm{GeV}$. The LO predictions of PythiA and Sherpa give a good description of the data, except for $p_{\mathrm{T}}^{\text {jet-lead }}$ in the case of PyTHIA. The fixed-order NLO QCD calculations of JETPHOX, corrected for hadronisation and underlying event effects, and the multi-leg NLO QCD plus parton shower calculations of SHERPA describe the measured cross sections within the theoretical uncertainties, larger than the experimental ones, as shown in Figure 3. The comparison with these predictions that use different PDF sets, show that the description of the data achieved 

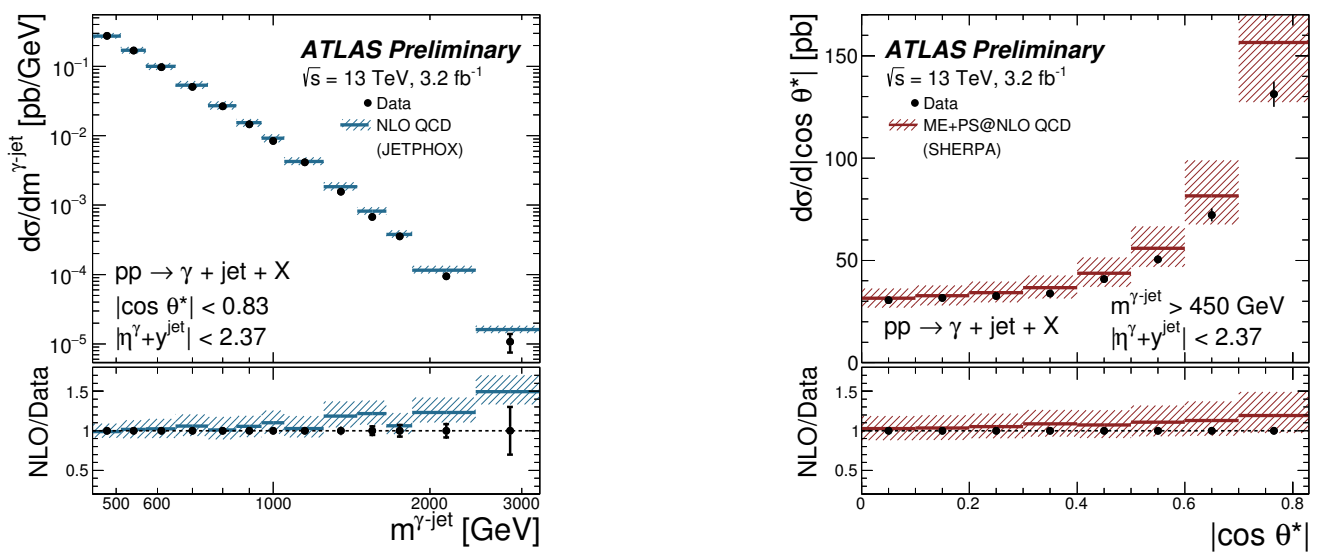

Figure 3. Measured cross section for isolated-photon plus a jet production (dots) as a function of the invariant mass of the photon-jet system, $m^{\gamma \text {-jet }}$ and of the scattering angle in the center-of-mass frame, compared with the fixed-order NLO QCD calculations of JETPHOx (left) and multi-leg NLO QCD plus parton shower predictions from SHERPA (right) [10]. The bottom part of both figures shows the ratio of the NLO QCD prediction to the measured cross section. The black error bars respresent the experimental uncertainties while the shaded bands are the theoretical uncertainties of the calculation.

does not depend on the used PDF set. The measured $d \sigma /\left|\cos \theta^{*}\right|$ is consistent with the dominance of processes in which a quark has been exchanged.

\subsection{Photon pair production}

Measurements of a photon pair production has been studied at a center-of-mass energy of $8 \mathrm{TeV}$ [11]. The leading and sub-leading photons are required to have $E_{\mathrm{T}}^{\gamma(1)}>40 \mathrm{GeV}$ and $E_{\mathrm{T}}^{\gamma(2)}>30 \mathrm{GeV}$ respectevely and $\left|\eta^{\gamma}\right|<2.37$ both. Differential cross sections are mesured as functions of the main variables of the photon pair system, together with the trasverse component of the diphoton tri-momentum respect to the thrust axis $\left(a_{\mathrm{T}}\right)$. The fixed-order QCD calculations of Diphox [12] and Ressos [13] at NLO, $2 \gamma$ NNLO at NNLO [14] are compared to the data. These predictions are unable to reproduce the measured cross sections: REsBos and DipHox miss higher orders, while the NNLO calculations of $2 \gamma$ NNLO improve the description, but are still insufficient to describe the data. The predictions of a parton-level calculation of varying the jet multiplicity up to $\mathrm{NLO}^{1}$, matched to the parton shower algorithm in SHERPA 2.2.1 provide a good description of the data. Figure 4 shows the comparison between the measured differential cross section as a function of $a_{\mathrm{T}}$ and the fiducial cross section compared to the different pQCD calculations.

\footnotetext{
${ }^{1}$ The $\gamma \gamma$ and $\gamma \gamma+1$ parton processes are generated at NLO accuracy, while $\gamma \gamma+2$ and 3 partons are generated at LO.
} 

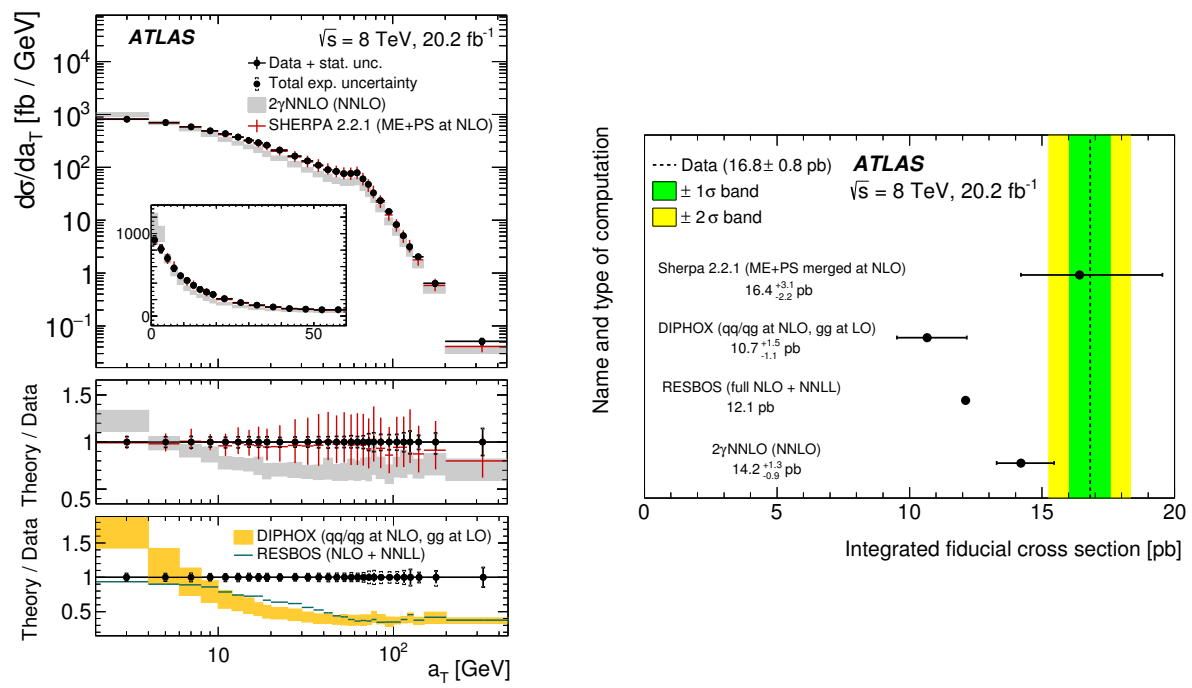

Figure 4. (Left) Differential cross section of the photon pair production as a function of $a_{\mathrm{T}}$ compared to the predictions of Diphox, ResBos, $2 \gamma$ NNLO and ShERPA 2.2.1. At the bottom of each plot, the ratio of the prediction to the data is shown. The ratio of Diphox and Resbos to the data is also shown. The bars and bands around the data and theoretical predictions represent the statistical and systematic uncertainties, estimated as described in the text [11]. Only the central values are shown for ResBos. (Right) Measured fiducial cross section compared to the predictions. The green (yellow) band represents the one- (two-)standard deviation uncertainty, including both the statistical and systematic uncertainties in the measurement added in quadrature.

\section{Jet production}

\subsection{Inclusive jet production at $8 \mathrm{TeV}$}

The inclusive jet cross-sections have been measured using the ATLAS $8 \mathrm{TeV}$ dataset [15]. Jets are reconstructed with the anti- $\mathrm{k}_{\mathrm{t}}$ algorithm with jet radius parameter values of $\mathrm{R}=0.4$ and $\mathrm{R}=0.6$, in the kinematic region of the jet transverse momentum from $p_{\mathrm{T}}>70 \mathrm{GeV}$ and $\left|y^{\text {jet }}\right|<3$. The cross sections are measured double-differentially in the jet transverse momentum and rapidity, as shown in Figure 5 (left). A fair agreement has been found in the comparison between the measured cross sections and the fixed-order NLO QCD calculations for different PDF sets, shown in Figure 5 (right), corrected for non-perturbative and electroweak effects, when considering the jet cross sections in individual jet rapidity bins treated independently. 

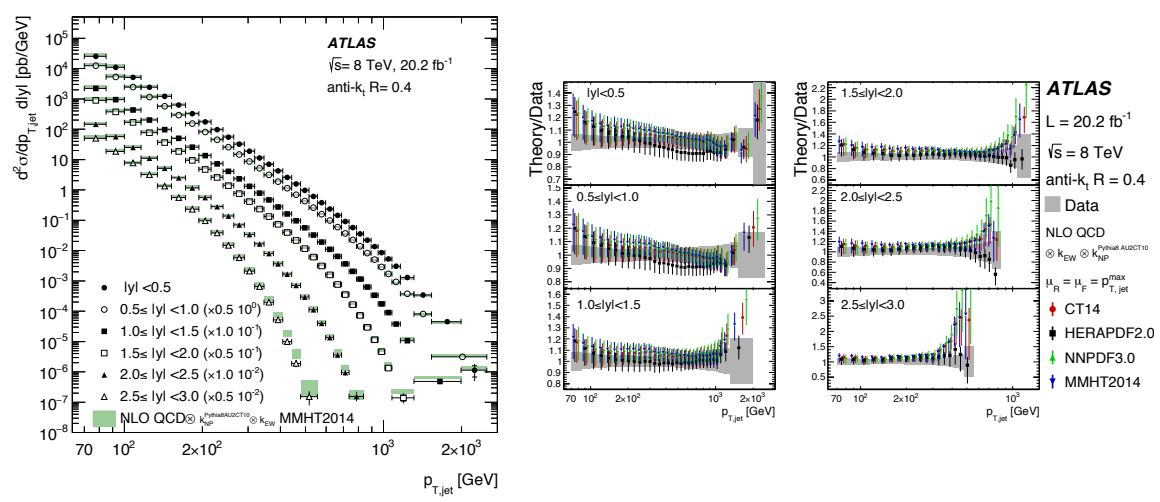

Figure 5. (Left) Inclusive jet cross-section at $8 \mathrm{TeV}$ as a function of jet $p_{\mathrm{T}}$ in bins of jet rapidity. The data are compared to the NLO QCD prediction with the MMHT2014 PDF set The error bars indicate the statistical uncertainty and the systematic uncertainty in the measurement added in quadrature. (Right) Ratio of the inclusive jet cross-section predicted by NLO QCD to the cross-section in data as a function of the jet $p_{\mathrm{T}}$ in each jet rapidity bin. Shown are the predictions for different PDF sets. The error bars indicate the total theory uncertainty. The grey band shows the total uncertainty in the measurement [15].

\subsection{Inclusive and dijet production at $13 \mathrm{TeV}$}

The inclusive jet and dijet cross sections have also been measured using the $3.2 \mathrm{fb}^{-1}$ ATLAS dataset at $13 \mathrm{TeV}$ [16]. The jets are reconstructed using the anti- $\mathrm{k}_{\mathrm{t}}$ with radius parameter $\mathrm{R}=0.4$. The cross sections were measured following the same method as for the $8 \mathrm{TeV}$ analysis. The double-differentially cross sections for the $13 \mathrm{TeV}$ measurement are shown in Figure 6 (left). NLO and NNLO pQCD calculations for the inclusive jet measurement, corrected for non-perturbative and electroweak effects, are compared to the measured cross sections. As for the $8 \mathrm{TeV}$ measurement, a good agreement has been found when considering the jet cross sections in individual jet rapidity bins independently. No significant deviations between the inclusive jet cross sections and the fixed order NNLO QCD calculations, corrected for non-perturbative and electroweak effects, are observed when using the transverse momentum of each jet, $p_{\mathrm{T}}^{\text {jet }}$, as QCD scale, as shown in Figure 6 (right). 

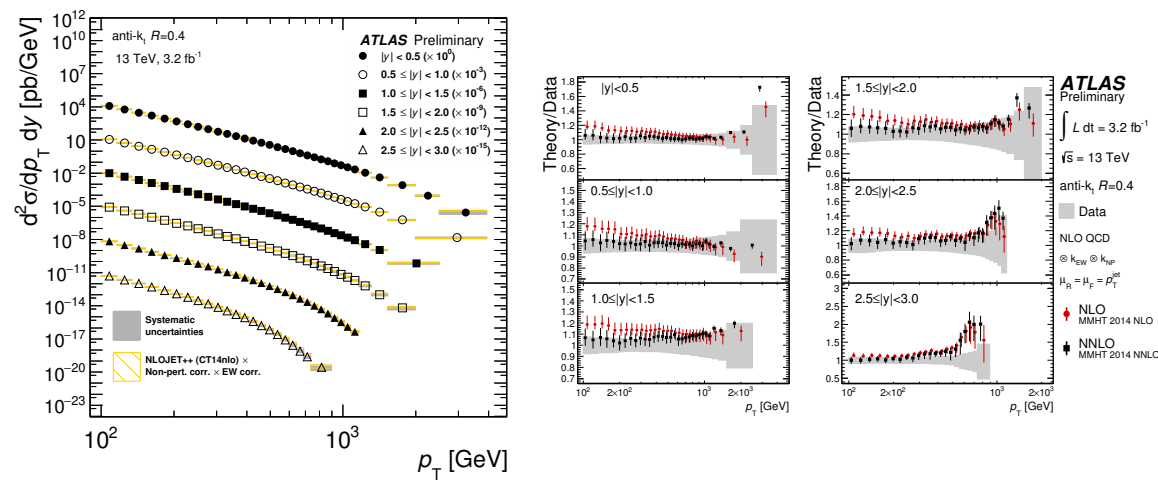

Figure 6. (Left) Inclusive jet cross-section at $13 \mathrm{TeV}$ as a function of jet $p_{\mathrm{T}}$ in bins of jet rapidity. The data are compared to the NLO QCD prediction with the MMHT2014 PDF set The error bars indicate the statistical uncertainty and the systematic uncertainty in the measurement added in quadrature [16]. (Right) Ratio of the inclusive jet cross-section predicted by NLO (red) and NNLO (black) QCD predictions to the cross-section in data as a function of the jet $p_{\mathrm{T}}$ in each jet rapidity bin. The error bars indicate the total theory uncertainty. The grey band shows the total uncertainty in the measurement.

In Figure 7 (left), the double-differential dijet production cross sections are presented as a function of the dijet invariant mass from $300 \mathrm{GeV}$ to $9 \mathrm{TeV}$ and half absolute rapidity separation between the two leading jets $\left(y^{*}\right)$, up to $y^{*}<3$. A fair agreement has been found in the comparison between the
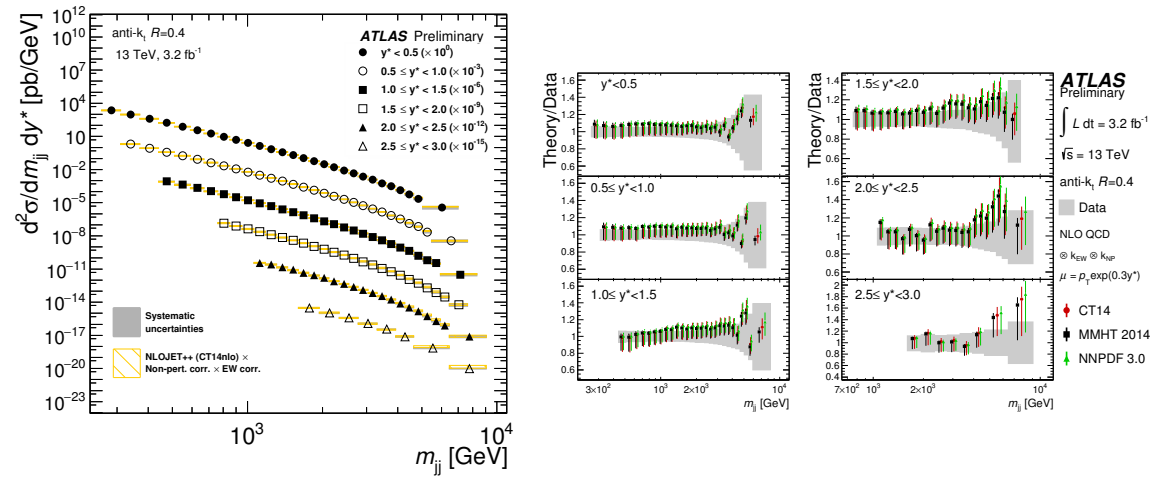

Figure 7. (Left) Dijet cross-sections as a function of $m_{\mathrm{jj}}$ and $y^{*}$, for anti- $k_{t}$ jets with $\mathrm{R}=0.4$. The shaded areas indicate the experimental systematic uncertainties. The data are compared to NLO pQCD predictions calculated using NLOJET++ with the CT14 NLO PDF set. The open boxes indicate the predictions with their uncertainties [16]. (Right) Ratio of NLO pQCD predictions to the measured dijet cross-sections. These ratios are shown as a function of the $m_{\mathrm{jj}}$ in six $\mathrm{y}^{*}$ bins. The predictions calculated using different PDF sets (MMHT2014 and NNPDF 3.0) are also shown. Their uncertainties are shown by the color lines. The grey band shows the uncertainty including both, systematic and statistical uncertainties. 
measured cross sections and the fixed-order NLO QCD calculations for different PDF sets, shown in Figure 7 (right), corrected for non-perturbative and electroweak effects.

\section{Transverse energy-energy correlations and $\alpha_{\mathrm{s}}$ extraction}

Transverse energy-energy correlations and the relative asymmetries in multi-jet events have been measured using the $8 \mathrm{TeV}$ ATLAS dataset [17]. The data were binned in six intervals of the scalar sum of the transverse momenta of the two leading jets, $H_{\mathrm{T} 2}=p_{\mathrm{T} 1}+p_{\mathrm{T} 2}$ and compared to the NLO pQCD predictions, corrected for hadronisation and multi-parton interaction effects. The comparison shows that the data are compatible with the theoretical predictions, within the uncertainties. The results are then used to determine the strong coupling constant $\alpha_{\mathrm{s}}$ and its evolution with a chosed scale $Q=H_{\mathrm{T} 2} / 2$, by means of a $\chi^{2}$ fit to the theoretical predictions for both TEEC and ATEEC in each energy bin. The results of the $\chi^{2}$ fit, together with other experiment determinations are shown in Figure 8. Moreover, global fits to the TEEC and ATEEC data are performed, obtaining

$$
\begin{aligned}
& \alpha_{\mathrm{s}}\left(m_{\mathrm{Z}}\right)=0.1162 \pm 0.0011(\text { exp. })_{-0.0061}^{+0.0076}(\text { scale }) \pm 0.0018(\mathrm{PDF}) \pm 0.0003(\mathrm{NP}) \\
& \alpha_{\mathrm{s}}\left(m_{\mathrm{Z}}\right)=0.1196 \pm 0.0013(\text { exp. })_{-0.0013}^{+0.0061}(\text { scale }) \pm 0.0017(\mathrm{PDF}) \pm 0.0004(\mathrm{NP})
\end{aligned}
$$

respectively. The two values are in good agreement with the worls average $\alpha_{\mathrm{s}}\left(m_{\mathrm{Z}}\right)=0.1181 \pm 0.0011$. The presented results are limited by the theoretical scale uncertainties, which amounts to the $6 \%$ (4 $\%$ ) of the value of $\alpha_{\mathrm{s}}\left(m_{\mathrm{Z}}\right)$ in the case of the TEEC (ATEEC). This uncertainty is expected to decrease as higher orders are calculated for the perturbative expansion.
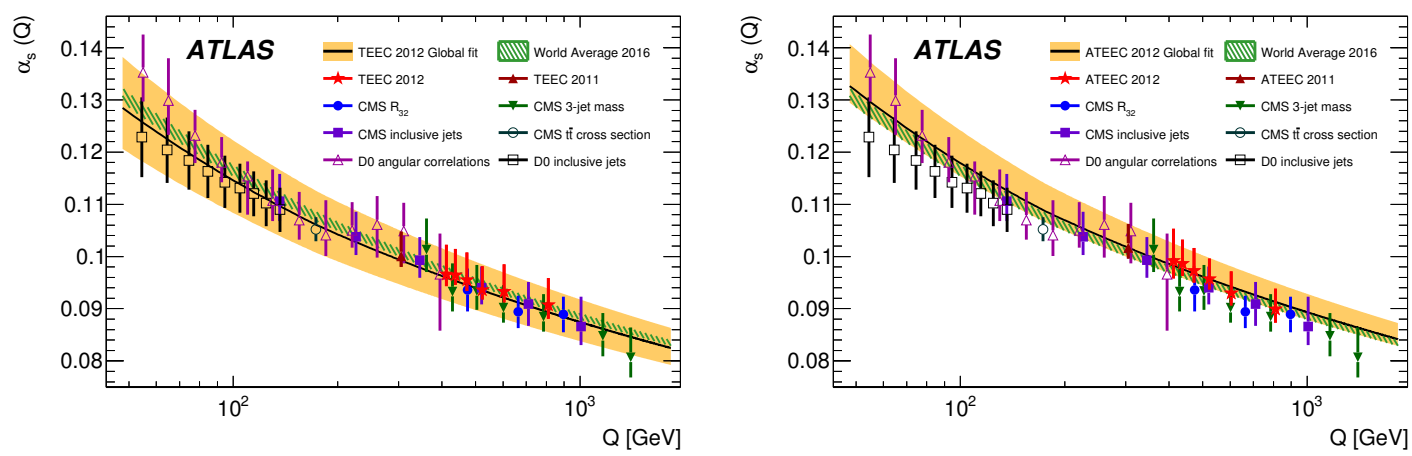

Figure 8. Comparison of the values of $\alpha_{\mathrm{s}}(\mathrm{Q})$ obtained from fits to the TEEC (left) and the ATEEC (right) functions at the energy scales given by $H_{\mathrm{T} 2} / 2$ (red star points) with the uncertainty band from the global fit (orange full band) and the 2016 world average (green hatched band). Determinations from other experiments are also shown as data points. The error bars, as well as the orange full band, include all experimental and theoretical sources of uncertainty. The strong coupling constant is assumed to run according to the two-loop solution of the renormalisation group equation [17].

\section{Summary}

In this paper high-precision measurements involving photons and jets at $8 \mathrm{TeV}$ are presented together with the first results at $13 \mathrm{TeV}$. All the mesurements are in good agreement with the pQCD predictions 
within the theoretical uncertainties, expecially those coming from missing higher order terms in the calculations. For the future, the ATLAS collaboration plans to improve these studies with higher statistics in order to explore the new energy regime opened by the LHC at $13 \mathrm{TeV}$ and to provide valuable physics inputs to PDF and $\alpha_{\mathrm{s}}$ fits.

\section{References}

[1] T. Pietrycki and A. Szczurek, Photon-jet correlations in $p p$ and $p \bar{p}$ collisions, Phys. Rev. D 76, 034003 (2007).

[2] ATLAS Collaboration, The ATLAS Experiment at the CERN Large Hadron Collider, JINST 3 (2008) S08003.

[3] ATLAS Collaboration, Measurement of the cross section for inclusive isolated-photon production in pp collisions at $\sqrt{s}=13 \mathrm{TeV}$ using the ATLAS detector, Phys. Lett. B 770 (2017) 473.

[4] T. Sjostrand, S. Mrenna and P. Z. Skands, A Brief Introduction to PYTHIA 8.1, Commun. 178 (2008) 852, arXiv:0710.3820.

[5] T. Gleisberg et al., Event generation with SHERPA 1.1, JHEP 02 (2009), p. 7. 10.1088/11266708/2009/02/007.

[6] S. Catani, M. Fontannaz, J. Ph. Guillet and E. Pilon, Cross section of isolated prompt photons in hadron-hadron collisions, arXiv:hep-ph/0204023.

[7] ATLAS Collaboration,High- $E_{\mathrm{T}}$ isolated-photon plus jets production in pp collisions at $\sqrt{s}=8$ TeV with the ATLAS detector, Nucl. Phys. B 918 (2017) 257.

[8] S. Catani, Y.L. Dokshitzer, M.H. Seymour and B.R. Webber, Longitudinally invariant Kt clustering algorithms for hadron hadron collisions, Nucl. Phys. B 406 (1993) 187.

[9] C.F. Berger et al., An Automated Implementation of On-Shell Methods for One-Loop Amplitudes, Phys. Rev. D 78 (2008) 036003.

[10] ATLAS Collaboration, First measurement of isolated-photon plus jet production in pp collisions at $\sqrt{s}=13 \mathrm{TeV}$ with the ATLAS detector, ATLAS-CONF-2017-059, https://atlas.web.cern.ch/Atlas/GROUPS/PHYSICS/CONFNOTES/ATLAS-CONF-2017-059/.

[11] ATLAS Collaboration, Measurements of integrated and differential cross sections for isolated photon pair production in pp collisions at $\sqrt{s}=8 \mathrm{TeV}$ with the ATLAS detector, Phys. Rev. D 95 (2017) 112005.

[12] T. Binoth, J. P. Guillet, E. Pilon and M. Werlen, A Full next-to-leading order study of direct photon pair production in hadronic collisions, Eur. Phys. J. C 16 (2000) 311.

[13] C. Balaza, E. Berger, P. Nadolsky and C.-P. Yuan, All-orders resummation for diphoton production at hadron colliders, Phys. Lett. B 637 (2006) 235.

[14] S. Catani, L. Cieri, D. de Florian, G. Ferrera and M. Grazzini, Diphoton production at hadron colliders: a fully-differential QCD calculation at NNLO, Phys. Rev. Lett. 108 (2012) 072001.

[15] ATLAS Collaboration, Measurement of the inclusive jet cross-sections in proton-proton collisions at $\sqrt{s}=8 \mathrm{TeV}$ with the ATLAS detector, JHEP 09 (2017) 020.

[16] ATLAS Collaboration, Measurement of inclusive-jet and dijet cross-sections in proton-proton collisions at $13 \mathrm{TeV}$ centre-of-mass energy with the ATLAS detector, ATLAS-CONF-2017-048, https://atlas.web.cern.ch/Atlas/GROUPS/PHYSICS/CONFNOTES/ATLAS-CONF-2017-048.

[17] ATLAS Collaboration, Determination of the strong coupling constant $\alpha_{s}$ from transverse energyenergy correlations in multijet events at $\sqrt{s}=8 \mathrm{TeV}$ using the ATLAS detector, arXiv:1707.02562 